\title{
An Algebraic Study of Gauss-Kronrod Quadrature Formulae for Jacobi Weight Functions*
}

\author{
By Walter Gautschi and Sotirios E. Notaris
}

\begin{abstract}
We study Gauss-Kronrod quadrature formulae for the Jacobi weight function $w^{(\alpha, \beta)}(t)=(1-t)^{\alpha}(1+t)^{\beta}$ and its special case $\alpha=\beta=\lambda-\frac{1}{2}$ of the Gegenbauer weight function. We are interested in delineating regions in the $(\alpha, \beta)$-plane, resp. intervals in $\lambda$, for which the quadrature rule has (a) the interlacing property, i.e., the Gauss nodes and the Kronrod nodes interlace; (b) all nodes contained in $(-1,1)$; (c) all weights positive; (d) only real nodes (not necessarily satisfying (a) and/or (b)). We determine the respective regions numerically for $n=1(1) 20(4) 40$ in the Gegenbauer case, and for $n=1(1) 10$ in the Jacobi case, where $n$ is the number of Gauss nodes. Algebraic criteria, in particular the vanishing of appropriate resultants and discriminants, are used to determine the boundaries of the regions identifying properties (a) and (d). The regions for properties (b) and (c) are found more directly. A number of conjectures are suggested by the numerical results. Finally, the Gauss-Kronrod formula for the weight $w^{(\alpha, 1 / 2)}$ is obtained from the one for the weight $w^{(\alpha, \alpha)}$, and similarly, the Gauss-Kronrod formula with an odd number of Gauss nodes for the weight function $w(t)=|t|^{\gamma}\left(1-t^{2}\right)^{\alpha}$ is derived from the Gauss-Kronrod formula for the weight $w^{(\alpha,(1+\gamma) / 2)}$.
\end{abstract}

1. Introduction. A Gauss-Kronrod quadrature formula for the (nonnegative) weight function $w$ on $[a, b]$ is a quadrature formula of the form

$$
\int_{a}^{b} f(t) w(t) d t=\sum_{\nu=1}^{n} \sigma_{\nu} f\left(\tau_{\nu}\right)+\sum_{\mu=1}^{n+1} \sigma_{\mu}^{*} f\left(\tau_{\mu}^{*}\right)+R_{n}(f)
$$

where $\tau_{\nu}=\tau_{\nu}^{(n)}$ are the Gaussian nodes (i.e., the zeros of $\pi_{n}(\cdot ; w d t)$, the $n$th degree (monic) orthogonal polynomial relative to the measure $d \sigma(t)=w(t) d t$ on $[a, b]$ ) and the nodes $\tau_{\mu}^{*}=\tau_{\mu}^{(n) *}$ (the "Kronrod nodes") and weights $\sigma_{\nu}=\sigma_{\nu}^{(n)}, \sigma_{\mu}^{*}=\sigma_{\mu}^{(n) *}$ are determined such that (1.1) has maximum degree of exactness $3 n+1$, i.e.,

$$
R_{n}(f)=0, \quad \text { all } f \in \mathbf{P}_{3 n+1} .
$$

It is well known that $\tau_{\mu}^{*}$ must be the zeros of the (monic) polynomial $\pi_{n+1}^{*}$ of degree $n+1$ orthogonal to all polynomials of degree $n$ with respect to the "weight function"

$$
w^{*}(t)=\pi_{n}(t ; w d t) w(t) \text { on }[a, b] .
$$

Even though $\pi_{n}$, and hence $w^{*}$, changes sign on $[a, b]$, it is known that $\pi_{n+1}^{*}$ exists uniquely (see, e.g., Gautschi [3, Section 3.1.2]). There is no guarantee, however, that the zeros $\tau_{\mu}^{*}$ of $\pi_{n+1}^{*}$ are inside the interval $[a, b]$, or real, for that matter.

Received June 18, 1987.

1980 Mathematics Subject Classification (1985 Revision). Primary 65D32; Secondary 33A65.

Key words and phrases. Gauss-Kronrod quadrature formulae, orthogonal polynomials.

*This work was supported, in part, by the National Science Foundation under grant DCR8320561. 
Our interest here is indeed in obtaining precise information about the reality and location of these zeros, as well as the positivity of all weights. We are studying these questions in the case where $w$ is the Jacobi weight function,

$$
w^{(\alpha, \beta)}(t)=(1-t)^{\alpha}(1+t)^{\beta}, \quad-1<t<1, \alpha>-1, \beta>-1,
$$

or its special case, the Gegenbauer weight

$$
w_{\lambda}(t)=w^{(\lambda-1 / 2, \lambda-1 / 2)}(t), \quad-1<t<1, \lambda>-\frac{1}{2} .
$$

We say that the nodes of (1.1) interlace if they are all real and, when ordered decreasingly, satisfy

$$
-\infty<\tau_{n+1}^{*}<\tau_{n}<\tau_{n}^{*}<\cdots<\tau_{2}^{*}<\tau_{1}<\tau_{1}^{*}<\infty
$$

Our objective is, for each fixed $n=1,2,3, \ldots$, to determine domains of the parameters $\alpha, \beta$ and $\lambda$, respectively, in which either of the following properties holds:

(a) The nodes $\tau_{\nu}, \tau_{\mu}^{*}$ interlace.

(b) All nodes $\tau_{\nu}, \tau_{\mu}^{*}$, in addition to satisfying (1.6), are contained in $(-1,1)$, i.e., $-1<\tau_{n+1}^{*}$ and $\tau_{1}^{*}<1$.

(c) The nodes interlace and each weight $\sigma_{\nu}$ is positive. (The positivity of $\sigma_{\mu}^{*}$ is equivalent to the interlacing property; see Monegato [5, Theorem 1].)

(d) All nodes, without necessarily satisfying (a) and/or (b), are real.

To answer these questions, we start from the known fact (see, e.g., Gautschi [3, Section 2.1.2]) that all properties (a)-(d) hold for the Gegenbauer weight (1.5), or the Jacobi weight (1.4) with $\alpha=\beta=\lambda-\frac{1}{2}$, when $0<\lambda<1$. Moving the parameters $\alpha, \beta$, or $\lambda$, continuously away from this segment induces a continuous motion of the nodes $\tau_{\nu}, \tau_{\mu}^{*}$, which, initially, are constrained to move on the real line. The interlacing property breaks down the first time a node $\tau_{\mu}^{*}$ collides with a node $\tau_{\nu}$. The polynomials $\pi_{n}$ and $\pi_{n+1}^{*}$ then have a common zero, a fact that can be detected by determining when the resultant $R\left(\pi_{n}, \pi_{n+1}^{*}\right)$ of $\pi_{n}$ and $\pi_{n+1}^{*}$ vanishes (for the first time). When a collision occurs, the nodes $\tau_{\nu}, \tau_{\mu}^{*}$ involved most likely cross each other, so that there are now two Kronrod nodes captured between two Gauss nodes. Only now is it possible that two Kronrod nodes may collide, giving rise to a pair of complex Kronrod nodes. The occurrence of this event can be detected by determining the appropriate zero of the resultant $R\left(\pi_{n+1}^{*}, \pi_{n+1}^{* \prime}\right)$. This allows us to settle property (d). Properties (b) and (c) are easily dealt with by examining when (for the first time) $\left(\tau_{n+1}^{*}+1\right)\left(\tau_{1}^{*}-1\right)=0$, and $\sigma_{\nu}=0$ for some $\nu$, respectively.

In Section 2 we carry out this program for the Gegenbauer weight (1.5). The success of the calculations, particularly when $n$ is large, depends crucially on the resultants involved being computed in a stable manner. This is discussed in Section 3. In Section 4 we report on limited explorations for the case of the Jacobi weight (1.4). Section 5 presents analytic treatments of Gauss-Kronrod formulae for Jacobi weights with parameter $\beta=\frac{1}{2}$ and for the weight function $w(t)=|t|^{\gamma}\left(1-t^{2}\right)^{\alpha}$, $\alpha>-1, \gamma>-1$, on $(-1,1)$.

2. Gegenbauer Weights. We consider here the weight function (1.5), that is,

$$
w_{\lambda}(t)=\left(1-t^{2}\right)^{\lambda-1 / 2}, \quad-1<t<1, \lambda>-\frac{1}{2} .
$$


Using

$$
\begin{aligned}
& \int_{-1}^{1} t^{2 m}\left(1-t^{2}\right)^{\lambda-1 / 2} d t=\int_{0}^{1} x^{m-1 / 2}(1-x)^{\lambda-1 / 2} d x \\
& \quad=B\left(m+\frac{1}{2}, \lambda+\frac{1}{2}\right)=\frac{\Gamma\left(m+\frac{1}{2}\right) \Gamma\left(\lambda+\frac{1}{2}\right)}{\Gamma(m+\lambda+1)}, \quad m=0,1,2, \ldots,
\end{aligned}
$$

it is straightforward (though tedious, at times) to compute $\pi_{n}, \pi_{n+1}^{*}$ explicitly for the first few values of $n$. One obtains, for $n=1,2,3$ and 4 ,

$$
\begin{gathered}
\pi_{1}(t)=t, \quad \pi_{2}^{*}(t)=t^{2}-\frac{3}{2(\lambda+2)} \\
\pi_{2}(t)=t^{2}-\frac{1}{2(\lambda+1)}, \quad \pi_{3}^{*}(t)=t^{3}-\frac{3}{\lambda+3} t \\
\pi_{3}(t)=t^{3}-\frac{3}{2(\lambda+2)} t, \quad \pi_{4}^{*}(t)=t^{4}-\frac{5}{\lambda+4} t^{2}+\frac{5}{4} \frac{16-\lambda}{(\lambda+4)^{2}(\lambda+5)} \\
\pi_{4}(t)=t^{4}-\frac{3}{\lambda+3} t^{2}+\frac{3}{4(\lambda+2)(\lambda+3)} \\
\pi_{5}^{*}(t)=t^{5}-\frac{15}{2(\lambda+5)} t^{3}+\frac{15}{4} \frac{\lambda+20}{(\lambda+5)^{2}(\lambda+6)} t
\end{gathered}
$$

Likewise, using the formula (Monegato [5, Eq. (2.5)])

$$
\sigma_{\nu}^{(n)}=\lambda_{\nu}^{(n)}+\frac{\left\|\pi_{n}\right\|_{w}^{2}}{\pi_{n+1}^{*}\left(\tau_{\nu}^{(n)}\right) \pi_{n}^{\prime}\left(\tau_{\nu}^{(n)}\right)}
$$

where $\lambda_{\nu}^{(n)}$ are the Christoffel numbers (i.e., the weights in the Gaussian quadrature rule) and $\|\cdot\|_{w}$ the $L_{2}$-norm for the weight function (2.1), one obtains

$\left(2.4_{1}\right) \quad \sigma_{1}^{(1)}=\frac{2 \sqrt{\pi}}{3} \frac{\Gamma(\lambda+3 / 2)}{\Gamma(\lambda+2)}$

$$
\sigma_{1}^{(2)}=\sigma_{2}^{(2)}=\frac{3 \sqrt{\pi}}{2} \frac{\Gamma\left(\lambda+\frac{1}{2}\right)(\lambda+1)^{2}}{\Gamma(\lambda+1)(\lambda+2)(5 \lambda+3)}
$$

$$
\sigma_{1}^{(3)}=\sigma_{3}^{(3)}=\frac{5 \sqrt{\pi}}{3} \frac{\Gamma\left(\lambda+\frac{1}{2}\right)(\lambda+2)^{4}(2 \lambda+3)}{\Gamma(\lambda+2)(\lambda+3)\left(26 \lambda^{3}+153 \lambda^{2}+336 \lambda+160\right)},
$$

$$
\sigma_{2}^{(3)}=\frac{8 \sqrt{\pi}}{15} \frac{\Gamma(\lambda+5 / 2)\left(40+7 \lambda-2 \lambda^{2}\right)}{\Gamma(\lambda+4)(16-\lambda)}
$$

$$
\begin{gathered}
\sigma_{1}^{(4)}=\sigma_{4}^{(4)}=\frac{5 \sqrt{\pi}}{2} \times \\
\Gamma(\lambda+3 / 2)(\lambda+2)(\lambda+3)^{4}\left[(\lambda+5)(\lambda+6)(2 \lambda+3)+\omega(\lambda+2)\left(\lambda^{2}-15\right)\right] \\
\Gamma(\lambda+5) \omega(\omega+3)(2 \lambda-\omega+3)\left[5 \lambda^{4}+11 \lambda^{3}-109 \lambda^{2}-465 \lambda-450+\omega(\lambda+2)(\lambda+5)(\lambda+6)(3 \lambda+5)\right]
\end{gathered}
$$

where $\omega=[3(2 \lambda+3) /(\lambda+2)]^{1 / 2}$ in $\left(2.4_{4}\right)$.

For $n=1$, the Gauss-Kronrod rule is the 3-point Gauss rule and therefore satisfies properties (a)-(d) for all $\lambda>-\frac{1}{2}$. If $n=2$, Eq. (2.2 2 ) shows that (a) [hence also (d)] holds for all $\lambda>-\frac{1}{2}$ and (b) for all $\lambda>0$, while Eq. (2.42) shows that (c) holds for all $\lambda>-\frac{1}{2}$. We now discuss in detail the two cases $n=3$ and 
$n=4$ for which we have analytic expressions for all the quantities of interest. They are representative for the cases $n$ odd and $n$ even, respectively, to be discussed subsequently.

If $n=3$, the polynomials $\pi_{3}$ and $\pi_{4}^{*}$ have common zeros if and only if either $\pi_{4}^{*}(0)=0$ (in which case 0 is a common zero) or the polynomials

$$
\begin{aligned}
& \frac{1}{\sqrt{x}} \pi_{3}(\sqrt{x})=x-\frac{3}{2(\lambda+2)}=: p_{3}(x) \\
& \pi_{4}^{*}(\sqrt{x})=x^{2}-\frac{5}{\lambda+4} x+\frac{5}{4} \frac{16-\lambda}{(\lambda+4)^{2}(\lambda+5)}=: p_{4}^{*}(x)
\end{aligned}
$$

have a common zero. The former, by $\left(2.2_{3}\right)$, is true exactly for $\lambda=16$, whereas the latter is true precisely if the resultant of $p_{3}$ and $p_{4}^{*}$ vanishes,

$$
R\left(p_{3}, p_{4}^{*}\right)=\left|\begin{array}{ccc}
1 & -\frac{3}{2(\lambda+2)} & 0 \\
0 & 1 & -\frac{3}{2(\lambda+2)} \\
1 & -\frac{5}{\lambda+4} & \frac{5}{4} \frac{16-\lambda}{(\lambda+4)^{2}(\lambda+5)}
\end{array}\right|=0 .
$$

An easy calculation shows that

$$
R\left(p_{3}, p_{4}^{*}\right)=-\frac{1}{4}(\lambda+2)^{-2}(\lambda+4)^{-2}(\lambda+5)^{-1}\left(26 \lambda^{3}+153 \lambda^{2}+336 \lambda+160\right) .
$$

The cubic polynomial on the right has one real zero at $-.6447375 \ldots$ and a pair of conjugate complex zeros, hence is positive for all $\lambda>-\frac{1}{2}$. Therefore, $R\left(p_{3}, p_{4}^{*}\right)<0$ for all $\lambda>-\frac{1}{2}$. It follows that (a) is true precisely for $-\frac{1}{2}<\lambda<16$, there occurring a collision of nodes at the origin when $\lambda=16$, but no other collisions. Since the zeros of $\pi_{4}^{*}$ are symmetric with respect to the origin, $\pi_{4}^{*}$ has a double zero at the origin when $\lambda=16$, which splits into a pair of conjugate complex zeros as $\lambda$ increases beyond 16 . Indeed, the constant term of $p_{4}^{*}$, hence at least one of the zeros of $p_{4}^{*}$, becomes (and remains) negative, giving rise to a pair of complex zeros of $\pi_{4}^{*}$. Therefore, (d) is true exactly for $-\frac{1}{2}<\lambda \leq 16$. Property (b) is discussed most easily by noting that it is equivalent to $\pi_{4}^{*}(1)>0$. Indeed, $\tau_{1}^{*}<1$ clearly implies $\pi_{4}^{*}(1)>0$, while, conversely, $\pi_{4}^{*}(1)>0$ implies $\tau_{1}^{*}<1$ since otherwise, by the interlacing property, $\tau_{1}<1 \leq \tau_{1}^{*}$, meaning that $\pi_{4}^{*}(1) \leq 0$. Since, by $\left(2.2_{3}\right)$,

$$
\pi_{4}^{*}(1)=\frac{1}{4} \lambda(\lambda+4)^{-2}(\lambda+5)^{-1}(2 \lambda+3)(2 \lambda+13)
$$

we have property (b) precisely if $\lambda>0$. The cubic polynomial in the denominator of $\sigma_{1}^{(3)}$ [cf. $\left.\left(2.4_{3}\right)\right]$ being the same as the one in $\left(2.6_{3}\right)$, hence positive for all $\lambda>-\frac{1}{2}$, it follows that $\sigma_{1}^{(3)}>0$ for all $\lambda>-\frac{1}{2}$. Assuming $-\frac{1}{2}<\lambda<16$, we have, on the other hand, $\sigma_{2}^{(3)}>0$ if and only if $40+7 \lambda-2 \lambda^{2}>0$, i.e., $-\frac{1}{2}<\lambda<\frac{1}{4}(7+3 \sqrt{41})=$ $6.552343 \ldots$ This settles property $(\mathrm{c})$.

Now consider $n=4$. Since $\pi_{4}(0) \neq 0$, the origin is never a common zero of $\pi_{4}$ and $\pi_{5}^{*}$, and $\pi_{4}, \pi_{5}^{*}$ have a common zero if and only if the same is true for the polynomials $\pi_{4}(\sqrt{x})=: p_{4}(x)$ and $(\sqrt{x})^{-1} \pi_{5}^{*}(\sqrt{x})=: p_{5}^{*}(x)$. Using $\left(2.2_{4}\right)$, a 
somewhat lengthy computation gives

$$
\begin{aligned}
R\left(p_{4}, p_{5}^{*}\right)=- & \frac{9}{16}(\lambda+2)^{-2}(\lambda+3)^{-2}(\lambda+5)^{-4}(\lambda+6)^{-2} \\
& \times\left[29 \lambda^{6}+1273 \lambda^{5}+11904 \lambda^{4}+48385 \lambda^{3}\right. \\
& \left.+91925 \lambda^{2}+78000 \lambda+22500\right] .
\end{aligned}
$$

The polynomial in brackets has two real zeros at $-.580667 \ldots$ and $-32.863977 \ldots$ and two pairs of conjugate complex zeros. Consequently, $R\left(p_{4}, p_{5}^{*}\right)<0$ for all $\lambda>-\frac{1}{2}$, and property (a) [hence also (d)] holds for all $\lambda>-\frac{1}{2}$. From $\left(2.2_{4}\right)$ we find

$$
\pi_{5}^{*}(1)=\frac{1}{4}(\lambda+5)^{-2}(\lambda+6)^{-1} \lambda\left(4 \lambda^{2}+34 \lambda+25\right)
$$

where the quadratic has the two negative zeros $-.813068 \ldots$ and $-7.686931 \ldots$, hence remains positive for $\lambda>-\frac{1}{2}$. Property (b), i.e., $\pi_{5}^{*}(1)>0$, therefore holds precisely for $\lambda>0$. Another lengthy (but elementary) computation, based on $\left(2.4_{4}\right)$, shows that $\sigma_{1}^{(4)}$ is positive for all $\lambda>-\frac{1}{2}$, but $\sigma_{2}^{(4)}>0$ only if $\lambda<$ $51.7868606883 \ldots$, the unique positive root of $\lambda^{3}-47 \lambda^{2}-245 \lambda-150=0$. Thus, property (c) holds precisely if this last condition is satisfied.

The results for $1 \leq n \leq 4$ are summarized in Table 2.1, which shows the interval $\lambda_{n}^{p}<\lambda<\Lambda_{n}^{p}$ in which property $(p)$ holds, $p=a, b, c, d$. An extended table for $n=5(1) 20(4) 40$ is given as Table A.1 in the appendix.** The reasonings used to compute Table A.1 were similar to the ones explained in the cases $n=3$ and $n=4$, and are now briefly described.

TABLE 2.1

Property $(p)(p=a, b, c, d)$ for $1 \leq n \leq 4$.

\begin{tabular}{c|cc|cc|cc|cc}
$n$ & $\lambda_{n}^{a}$ & $\Lambda_{n}^{a}$ & $\lambda_{n}^{b}$ & $\Lambda_{n}^{b}$ & $\lambda_{n}^{c}$ & $\Lambda_{n}^{c}$ & $\lambda_{n}^{d}$ & $\Lambda_{n}^{d}$ \\
\hline 1 & $-\frac{1}{2}$ & $\infty$ & $-\frac{1}{2}$ & $\infty$ & $-\frac{1}{2}$ & $\infty$ & $-\frac{1}{2}$ & $\infty$ \\
2 & $-\frac{1}{2}$ & $\infty$ & 0 & $\infty$ & $-\frac{1}{2}$ & $\infty$ & $-\frac{1}{2}$ & $\infty$ \\
3 & $-\frac{1}{2}$ & 16 & 0 & 16 & $-\frac{1}{2}$ & $(7+3 \sqrt{41}) / 4$ & $-\frac{1}{2}$ & 16 \\
4 & $-\frac{1}{2}$ & $\infty$ & 0 & $\infty$ & $-\frac{1}{2}$ & $51.786 \ldots$ & $-\frac{1}{2}$ & $\infty$
\end{tabular}

It is convenient to distinguish between $n=2 m$ even, in which case we write

$\left(2.8_{\text {even }}\right)$

$$
\pi_{2 m}(\sqrt{x})=x^{m}+a_{2 m, 1} x^{m-1}+\cdots+a_{2 m, m}=: p_{2 m}(x)
$$

$$
\frac{1}{\sqrt{x}} \pi_{2 m+1}^{*}(\sqrt{x})=x^{m}+b_{2 m+1,1} x^{m-1}+\cdots+b_{2 m+1, m}=: p_{2 m+1}^{*}(x)
$$

and $n=2 m-1$ odd, in which case we write

$\left(2.8_{\text {odd }}\right)$

$$
\begin{aligned}
\frac{1}{\sqrt{x}} \pi_{2 m-1}(\sqrt{x}) & =x^{m-1}+a_{2 m-1,1} x^{m-2}+\cdots+a_{2 m-1, m-1} \\
& =: p_{2 m-1}(x), \\
\pi_{2 m}^{*}(\sqrt{x}) & =x^{m}+b_{2 m, 1} x^{m-1}+\cdots+b_{2 m, m}=: p_{2 m}^{*}(x) .
\end{aligned}
$$

** Professor I. P. Mysovskih informed the first-named author by letter of October 28, 1987, that L. N. Puolokaĭnen [7], in a 1964 diploma paper prepared under his guidance, obtained $\lambda_{n}^{a}=-\frac{1}{2}$ for $n=1(1) 7$ and the same values of $\Lambda_{n}^{a}, n=1(1) 4$, as shown in Table 2.1. She furthermore calculated $6 \mathrm{D}$ values of $\Lambda_{n}^{a}, n=5(1) 7$, which agree with ours in Table A.1 to 4-5 significant digits. 
(Computational details for generating the coefficients in (2.8) and for the procedures to be described will be discussed in Section 3.)

To examine property (a), note that in the case $n=2 m$, since $a_{2 m, m} \neq 0$, the polynomials $\pi_{2 m}$ and $\pi_{2 m+1}^{*}$ have a common zero if and only if $p_{2 m}$ and $p_{2 m+1}^{*}$ do, i.e., precisely if the resultant $R\left(p_{2 m}, p_{2 m+1}^{*}\right)$ vanishes. This resultant, of course, has a constant sign on the interval $0<\lambda<1$; in all cases computed, it was found that the sign on $-\frac{1}{2}<\lambda \leq 0$ remained the same. Consequently, $\lambda_{n}^{a}=-\frac{1}{2}$. The quantity $\Lambda_{n}^{a}$ is the first value of $\lambda$ for which the resultant vanishes. This value was determined by a preliminary search, followed by the bisection method. The case $n=2 m-1$ is handled similarly, except for the additional possibility that the origin is a common zero of $\pi_{2 m-1}$ and $\pi_{2 m}^{*}$. This was detected (if the case indeed occurs) by the coefficient $b_{2 m, m}$ changing its sign. Our numerical work suggests the following

CONJECTURE 2.1. The Kronrod nodes $\tau_{\mu}^{(n) *}$ and Gauss nodes $\tau_{\nu}^{(n)}$ for the weight function $w_{\lambda}$ in (2.1) interlace if $-\frac{1}{2}<\lambda<\Lambda_{n}^{a}$, where $\Lambda_{n}^{a}$ are certain constants $>1$. (For numerical values of $\Lambda_{n}^{a}, n=1(1) 20(4) 40$, see Tables 2.1 and A.1.)

Property (b), as in the cases $n=3,4$, is settled by determining the subinterval of $\left(-\frac{1}{2}, \Lambda_{n}^{a}\right)$ in which $\pi_{n+1}^{*}(1)>0$, and property (c) by determining the subinterval of $\left(-\frac{1}{2}, \Lambda_{n}^{a}\right)$ in which $\sigma_{\nu}^{(n)}>0$ for all $\nu=1,2, \ldots, n$. The results can be summarized as

CONJECTURE 2.2. The Kronrod nodes $\tau_{\mu}^{(n) *}$ and Gauss nodes $\tau_{\nu}^{(n)}$ for the weight function $w_{\lambda}$ in (2.1), in addition to interlacing, are all contained in $(-1,1)$ if $0<$ $\lambda<\Lambda_{n}^{a}$, where $\Lambda_{n}^{a}$ are the constants in Conjecture 2.1 ; some Kronrod nodes are outside of $[-1,1]$ if $\lambda<0$.

CONJECTURE 2.3. The Kronrod nodes and Gauss nodes for the weight function $w_{\lambda}$ in (2.1) interlace, and all weights $\sigma_{\nu}^{(n)}$ are positive, if $-\frac{1}{2}<\lambda<\Lambda_{n}^{c}$, where $\Lambda_{n}^{c}$ are certain constants $1<\Lambda_{n}^{c}<\Lambda_{n}^{a}$. (For numerical values of $\Lambda_{n}^{c}, n=1(1) 20(4) 40$, see Tables 2.1 and A.1.)

Property (d), finally, needs to be considered only for $\lambda \geq \Lambda_{n}^{a}$ and requires the examination of discriminants. Complex zeros (of $\pi_{n+1}^{*}$ ) indeed can only arise from multiple zeros, i.e., after $R\left(\pi_{n+1}^{*}, \pi_{n+1}^{* \prime}\right)$ has vanished. The discussion of this again depends, in part, on the parity of $n$. If $n=2 m$, we write

$$
\frac{1}{\sqrt{x}} \pi_{2 m+1}^{*}(\sqrt{x})=x^{m}+b_{2 m+1,1} x^{m-1}+\cdots+b_{2 m+1, m}=: p_{2 m+1}^{*}(x),
$$

$$
\begin{aligned}
\pi_{2 m+1}^{* \prime}(\sqrt{x}) & =(2 m+1) x^{m}+(2 m-1) b_{2 m+1,1} x^{m-1}+\cdots+b_{2 m+1, m} \\
& =: q_{2 m+1}^{*}(x),
\end{aligned}
$$

and if $n=2 m-1$,

$$
\begin{aligned}
\pi_{2 m}^{*}(\sqrt{x}) & =x^{m}+b_{2 m, 1} x^{m-1}+\cdots+b_{2 m, m}=: p_{2 m}^{*}(x), \\
\frac{1}{\sqrt{x}} \pi_{2 m}^{* \prime}(\sqrt{x}) & =2 m x^{m-1}+(2 m-2) b_{2 m, 1} x^{m-2}+\cdots+2 b_{2 m, m-1} \\
& =: q_{2 m}^{*}(x) .
\end{aligned}
$$

There are two possibilities: Either the common zero of $\pi_{n+1}^{*}$ and $\pi_{n+1}^{* \prime}$ is also a zero of $\pi_{n}$, or it is not. The first case can only occur if two Kronrod nodes collide with one another and simultaneously with a Gauss node. If $n$ is even, this 
is unlikely to occur and, in fact, was never observed. It is also unlikely, and was not observed, when $n$ is odd, unless the collision takes place at the origin,

$$
\pi_{n}(0)=\pi_{n+1}^{*}(0)=\pi_{n+1}^{* \prime}(0) \quad(n=2 m-1 \text { odd })
$$

in which case, since $\lambda \geq \Lambda_{n}^{a}$, we must have $\lambda=\Lambda_{n}^{a}$. The event (2.10) indeed seems to occur whenever $m$ in (2.10) is even; then, moreover, $b_{2 m, m}$ in (2.9 odd $)$ was observed to change sign from positive to negative (cf. the discussion of property (a) above). This means that for $\lambda$ immediately beyond $\Lambda_{n}^{a}$, the polynomial $p_{2 m}^{*}$ has at least one negative zero (since $m$ is even!), hence $\pi_{2 m}^{*}$ a pair of conjugate complex zeros. Consequently, $\Lambda_{n}^{d}=\Lambda_{n}^{a}$ in this case.

If the common zero of $\pi_{n+1}^{*}$ and $\pi_{n+1}^{* \prime}$ is not a zero of $\pi_{n}$, then necessarily $\Lambda_{n}^{d}>\Lambda_{n}^{a}$. It was found, then, that $b_{2 m+1, m}$ and $b_{2 m, m}$ in (2.9) do not vanish, so that the zero in question cannot be the origin. It then follows that $\pi_{n+1}^{*}$ and $\pi_{n+1}^{* \prime}$ have a common zero if and only if $p_{n+1}^{*}$ and $q_{n+1}^{*}$ do, i.e., if $R\left(p_{n+1}^{*}, q_{n+1}^{*}\right)=0$. This event again can be determined by a search and bisection procedure. It transpired that the resultant $R\left(p_{n+1}^{*}, q_{n+1}^{*}\right)$ not only vanishes for some $\lambda=\lambda^{*}>\Lambda_{n}^{a}$, but also changes sign there. Since

$$
R\left(p_{n+1}^{*}, q_{n+1}^{*}\right)=\prod_{\mu} p_{n+1}^{*}\left(\xi_{\mu}\right)
$$

where $\xi_{\mu}$ are the zeros of $q_{n+1}^{*}$ (cf. [10, Section 5.9]), a pair of positive zeros of $p_{n+1}^{*}$ (and hence a pair of real zeros of $\pi_{n+1}^{*}$ ) coalesce and then disappear as $\lambda$ passes through $\lambda^{*}$, i.e., $p_{n+1}^{*}$ (and hence $\pi_{n+1}^{*}$ ) has a pair of conjugate complex zeros for $\lambda$ immediately beyond $\lambda^{*}$. There follows $\lambda^{*}=\Lambda_{n}^{d}$. Thus we form the

CONJECTURE 2.4. All Kronrod nodes $\tau_{\mu}^{(n) *}$ for the weight function $w_{\lambda}$ in (2.1) are real if $-\frac{1}{2}<\lambda \leq \Lambda_{n}^{d}$, where $\Lambda_{n}^{d}(n \neq 1,2,4)$ are certain constants either slightly larger than $\Lambda_{n}^{a}$, or equal to $\Lambda_{n}^{a}$, the latter precisely if $n=4 r-1, r=1,2,3, \ldots$. (For numerical values of $\Lambda_{n}^{d}, n=1(1) 20(4) 40$, see Tables 2.1 and A.1.)

3. Computational Considerations. All computations were performed on the CDC 6500 computer in single or double precision (machine precision $3.55 \times 10^{-15}$ and $1.26 \times 10^{-29}$, respectively).

Two different methods were used to compute the various resultants involved. One is an obvious extension of the method exemplified in Section 2. The coefficients $a_{n, k}$ of the polynomial $p_{n}$ (cf. (2.8)) are first computed by a recurrence relation that results from the linear relation connecting three consecutive $\pi_{k}$ of the same parity. The coefficients $b_{n, k}$ of $p_{n+1}^{*}$ (cf. (2.8)) then satisfy a system of linear algebraic equations expressing orthogonality of $\pi_{n+1}^{*}$ (with respect to the weight function $\left.w^{*}=w_{\lambda} \pi_{n}\right)$ to the first $[(n+1) / 2]$ odd powers. This system has been solved using the LINPACK [2] routines SGECO, SGESL (and their double-precision companions), whereupon the resultants of $p_{n}$ and $p_{n+1}^{*}$ and of $p_{n+1}^{*}$ and $q_{n+1}^{*}$ can be computed in determinant form (cf. [10, Section 5.8, Eq. (5.20)]), using again the factoring routine SGECO and its double-precision version. The major weakness of this approach is the severe ill-conditioning of the determinants involved. Their condition numbers (as estimated by SGECO) range from about $10^{5}$ for $n=6$ to about $10^{16}$ for $n=20$, precluding the safe use of our procedure in single precision, and also its use in double precision much beyond $n=20$. 
To avoid (or at least alleviate) this problem of ill-conditioning, we express the polynomial $\pi_{n+1}^{*}$ in terms of Gegenbauer polynomials and compute the expansion coefficients from a triangular system of equations as described in [1, Section 4], using Gauss-Jacobi quadrature to generate the matrix elements. If $n=2 m$ is even, we then have [10, Section 5.9]

$$
\left(3.1_{\text {even }}\right) \quad R\left(p_{2 m}, p_{2 m+1}^{*}\right)=\prod_{\mu=1}^{m} p_{2 m+1}^{*}\left(\tau_{\mu}^{2}\right)=\prod_{\mu=1}^{m}\left[\frac{1}{\tau_{\mu}} \pi_{2 m+1}^{*}\left(\tau_{\mu}\right)\right]
$$

and, if $n=2 m-1$,

$$
R\left(p_{2 m-1}, p_{2 m}^{*}\right)=\prod_{\mu=1}^{m-1} p_{2 m}^{*}\left(\tau_{\mu}^{2}\right)=\prod_{\mu=1}^{m-1} \pi_{2 m}^{*}\left(\tau_{\mu}\right)
$$

where $\tau_{\nu}=\tau_{\nu}^{(n)}$ are the zeros of $\pi_{n}$ in decreasing order, $\tau_{1}>\tau_{2}>\cdots>\tau_{n}$. Each factor in (3.1) is evaluated by Clenshaw's algorithm.

Similarly, the resultant of $p_{n+1}^{*}$ and $q_{n+1}^{*}$, required to analyze property (d), is computed for even $n=2 m$ by

$\left(3.2_{\text {even }}\right)$

$$
R\left(p_{2 m+1}^{*}, q_{2 m+1}^{*}\right)=\prod_{\mu=1}^{m} p_{2 m+1}^{*}\left(\tau_{\mu}^{\prime 2}\right)=\prod_{\mu=1}^{m}\left[\frac{1}{\tau_{\mu}^{\prime}} \pi_{2 m+1}^{*}\left(\tau_{\mu}^{\prime}\right)\right]
$$

and for odd $n=2 m-1$ by

$$
R\left(p_{2 m}^{*}, q_{2 m}^{*}\right)=\prod_{\mu=1}^{m-1} p_{2 m}^{*}\left(\tau_{\mu}^{\prime 2}\right)=\prod_{\mu=1}^{m-1} \pi_{2 m}^{*}\left(\tau_{\mu}^{\prime}\right)
$$

where $\tau_{1}^{\prime}>\tau_{2}^{\prime}>\cdots>\tau_{n}^{\prime}$ are the zeros of $\pi_{n+1}^{* \prime}$. To compute these zeros, we used, for the initial value $\lambda \approx \Lambda_{n}^{a}$ of $\lambda$, a simple search procedure followed by Newton's method. Then, as $\lambda$ was incremented by small amounts, and during the bisection procedure for determining $\Lambda_{n}^{d}$, the zeros found for one $\lambda$ were used as initial approximations for computing the zeros for the next $\lambda$ by Newton's method. The factors in (3.2) again were evaluated by Clenshaw's algorithm.

The computations based on (3.1), (3.2) appear to produce rather accurate results, even for relatively large values of $n$. For example, when $n=40$, we still obtained 10 correct decimal digits in single precision, as was confirmed by recomputation in double precision.

4. Jacobi Weights. We now consider property $(p), p=a, b, c$, for the general Jacobi weight $w^{(\alpha, \beta)}$ in (1.4). (Property (d) was not investigated, except for $n=1$, since the effort involved seemed to us excessive, given the chance that the curve $d$ could be indistinguishable from the curve $a$; cf. Table A.1 and Figure 4.1.) Noting that $w^{(\alpha, \beta)}(-t)=w^{(\beta, \alpha)}(t)$ and recalling the well-known fact that $\pi_{n}^{(\beta, \alpha)}(t)=$ $(-1)^{n} \pi_{n}^{(\alpha, \beta)}(-t)$, it is easy to show that

$$
\pi_{n+1}^{*(\beta, \alpha)}(t)=(-1)^{n+1} \pi_{n+1}^{*(\alpha, \beta)}(-t)
$$

and

$$
\begin{array}{ll}
\sigma_{\nu}^{(\beta, \alpha)}=\sigma_{n+1-\nu}^{(\alpha, \beta)}, & \nu=1,2, \ldots, n \\
\sigma_{\mu}^{*(\beta, \alpha)}=\sigma_{n+2-\mu}^{*(\alpha, \beta)}, & \mu=1,2, \ldots, n+1 .
\end{array}
$$


Interchanging $\alpha$ and $\beta$, therefore, has no effect on the validity of property $(p)$, $p=a, b, c$. It thus suffices to consider $\beta \geq \alpha$.

The case $n=1$ can be handled analytically. One finds

$$
R\left(\pi_{1}, \pi_{2}^{*}\right)=-\frac{4}{\alpha+\beta+3}\left\{\frac{(\alpha+1)(\beta+1)}{(\alpha+\beta+2)^{2}}+\frac{2(\alpha+2)(\beta+2)(\alpha+\beta+2)}{(\alpha+\beta+4)^{2}(\alpha+\beta+5)}\right\}<0
$$

so that property (a), hence also property (d), holds for all $\alpha>-1, \beta>-1$. The same is true for property (c), since

$$
\begin{aligned}
\sigma_{1}= & \frac{2^{\alpha+\beta+1} \Gamma(\alpha+2) \Gamma(\beta+2)}{\Gamma(\alpha+\beta+2)} \\
& \cdot\left\{\frac{1}{(\alpha+1)(\beta+1)}-\frac{1}{(\alpha+1)(\beta+1)+\frac{2(\alpha+2)(\beta+2)(\alpha+\beta+2)^{3}}{(\alpha+\beta+4)^{2}(\alpha+\beta+5)}}\right\}>0 .
\end{aligned}
$$

For property (b) we must show $\pi_{2}^{*}(1)>0$ and $\pi_{2}^{*}(-1)>0$. A simple calculation gives

$$
\pi_{2}^{*}(1)=\frac{4(\alpha+2)\left(\alpha^{2}+\alpha \beta+7 \alpha-\beta+4\right)}{(\alpha+\beta+4)^{2}(\alpha+\beta+5)},
$$

which is positive (for $\alpha>-1, \beta>-1$ ) precisely if

$$
\beta(\alpha-1)>-\left(\alpha^{2}+7 \alpha+4\right) \text {. }
$$

For $\alpha>1$, this inequality is true (since $\beta>-1$ ), while for $-1<\alpha<1$ it is true when

$$
\beta<\frac{\alpha^{2}+7 \alpha+4}{1-\alpha}, \quad-1<\alpha<1 \text {. }
$$

This defines a curve in the $(\alpha, \beta)$-plane that starts at the point $(-1,-1)$ and increases monotonically until it reaches a vertical asymptote at $\alpha=1$. By (4.1), one has the same expression as in (4.5) for $\pi_{2}^{*}(-1)$, except that $\alpha$ and $\beta$ are interchanged.

We summarize as follows: For $n=1$, the Gauss-Kronrod formula (1.1) for the Jacobi weight $w^{(\alpha, \beta)}$ satisfies properties (a), (c) and (d) for all $\alpha>-1, \beta>-1$, and property (b) precisely in the region

$$
\alpha \leq \beta<\frac{\alpha^{2}+7 \alpha+4}{1-\alpha}, \quad-1<\alpha<1,
$$

and in its symmetric image with respect to the diagonal $\alpha=\beta$. (In particular, property (b) holds for all $\alpha>1, \beta>1$.)

In order to delineate the regions of validity of property $(p)$ for values of $n$ larger than 1, we used procedures similar to those described in Section 2. Letting $\alpha$ move through the interval $\left(-1, \Lambda_{n}^{p}-\frac{1}{2}\right)$, for each $\alpha$ we started with $\beta=\alpha$ and increased $\beta$ in fixed (sufficiently small!) steps to determine the first change in the truth value of property $(p)$. Thereupon, the bisection method was used to narrow down the changeover point more accurately. The procedure had to be slightly modified for property (b), when $n$ is even, since there are two critical values of $\beta$ to be determined for $\alpha$ near and $\geq-\frac{1}{2}$. The smaller of the two was determined as before, the other by starting with $\beta=0$ (instead of $\beta=\alpha$ ). 
The validity of property (a) depends on the sign of the resultant $R\left(\pi_{n}, \pi_{n+1}^{*}\right)$, which was computed as in (3.1), except that symmetry could no longer be assumed; thus,

$$
R\left(\pi_{n}, \pi_{n+1}^{*}\right)=\prod_{\nu=1}^{n} \pi_{n+1}^{*}\left(\tau_{\nu}\right)
$$

Property (b) holds exactly if both of the inequalities

$$
\pi_{n+1}^{*}(1)>0, \quad(-1)^{n+1} \pi_{n+1}^{*}(-1)>0
$$

hold, while property (c) amounts to the positivity of all $\sigma_{\nu}^{(n)}$ in (2.3).

The results of our calculations are depicted graphically in Figure 4.1 for $n=$ $2(1) 10$. The region of validity for property $(p)$ is always located below the curve labeled $p$, except for the case $p=b, n$ even, $-1<\alpha<0$, where property (b) holds above (or to the right) of curve $b$.

Figure 4.1 suggests the validity of the following conjectures.

CONJECTURE 4.1. If $n$ is even, property (a) implies property (b) whenever $\alpha>$ $\alpha_{n}$, where $-\frac{1}{2}<\alpha_{n}<-.470, \alpha_{n} \rightarrow-\frac{1}{2}$ as $n \rightarrow \infty$.

CONJECTURE 4.2. If $n$ is odd, property (b) is false for $-1<\alpha<-\frac{1}{2}$.

The fact that property (b) is false for $n$ even, $\alpha=-\frac{1}{2},-\frac{1}{2}<\beta<\frac{1}{2}$, and for $n$ odd, $\alpha=-\frac{1}{2}, \frac{1}{2}<\beta<\frac{3}{2}$, is proved by Rabinowitz in [8, p. 75].**

Verification of properties (a) and (c), when $\alpha>0$, was found to be delicate at times, because of the resultant (for fixed $\alpha$ and varying $\beta$ ) exhibiting near double zeros, i.e., changing sign for two $\beta$-values very close together. For example, when $n=5$ and $\alpha=3.75$, a first change of sign of the resultant (4.8) from negative to positive was observed between $\beta=7.520$ and $\beta=7.521$, which was followed by a change from positive to negative between $\beta=7.540$ and $\beta=7.541$. The increment in $\beta$, therefore, had to be chosen sufficiently small to detect this change of sign. Such difficulties were observed typically near points where the slope of the curve $a$ or $c$ undergoes a rapid change (the "kinks" in the graphs for $a$ and $c$ of Figure 4.1).

5. Special Weights. Simple transformations allow us to reduce special Jacobi weights with $\beta=\frac{1}{2}$ to Gegenbauer weights and Gegenbauer weights multiplied by a power of $|t|$ to Jacobi weights. Some consequences of this for Gauss-Kronrod formulae will now be explored.

5.1. The Jacobi weight $w^{(\alpha, 1 / 2)}$. It is well known (see, e.g., [9, Eq. (4.1.5)]), and easily verified, that

$$
t \pi_{n}^{(\alpha, 1 / 2)}\left(2 t^{2}-1\right)=2^{n} \pi_{2 n+1}^{(\alpha, \alpha)}(t), \quad \alpha>-1 .
$$

We depart from the Gauss-Kronrod formula (assumed to exist)

$$
\int_{-1}^{1} f(t) w^{(\alpha, \alpha)}(t) d t=\sum_{\nu=1}^{2 n+1} \bar{\sigma}_{\nu} f\left(\bar{\tau}_{\nu}\right)+\sum_{\mu=1}^{2 n+2} \bar{\sigma}_{\mu}^{*} f\left(\bar{\tau}_{\mu}^{*}\right), \quad \text { all } f \in \mathbf{P}_{6 n+4}
$$

with $2 n+1$ Gauss nodes $\bar{\tau}_{\nu}=\tau_{\nu}^{(\alpha, \alpha)}$ and $2 n+2$ Kronrod nodes $\bar{\tau}_{\mu}^{*}$, ordered decreasingly as in (1.6); in particular,

$$
0<\bar{\tau}_{n+1}^{*}<\bar{\tau}_{n}<\cdots<\bar{\tau}_{1}<\bar{\tau}_{1}^{*}<1
$$

${ }^{* * *}$ The superscript $\mu+\frac{1}{2}$ in Eq. (68) of [8] should read $\mu-\frac{1}{2}$ (twice). The same change is required in the discussion immediately following Eq. (69). 

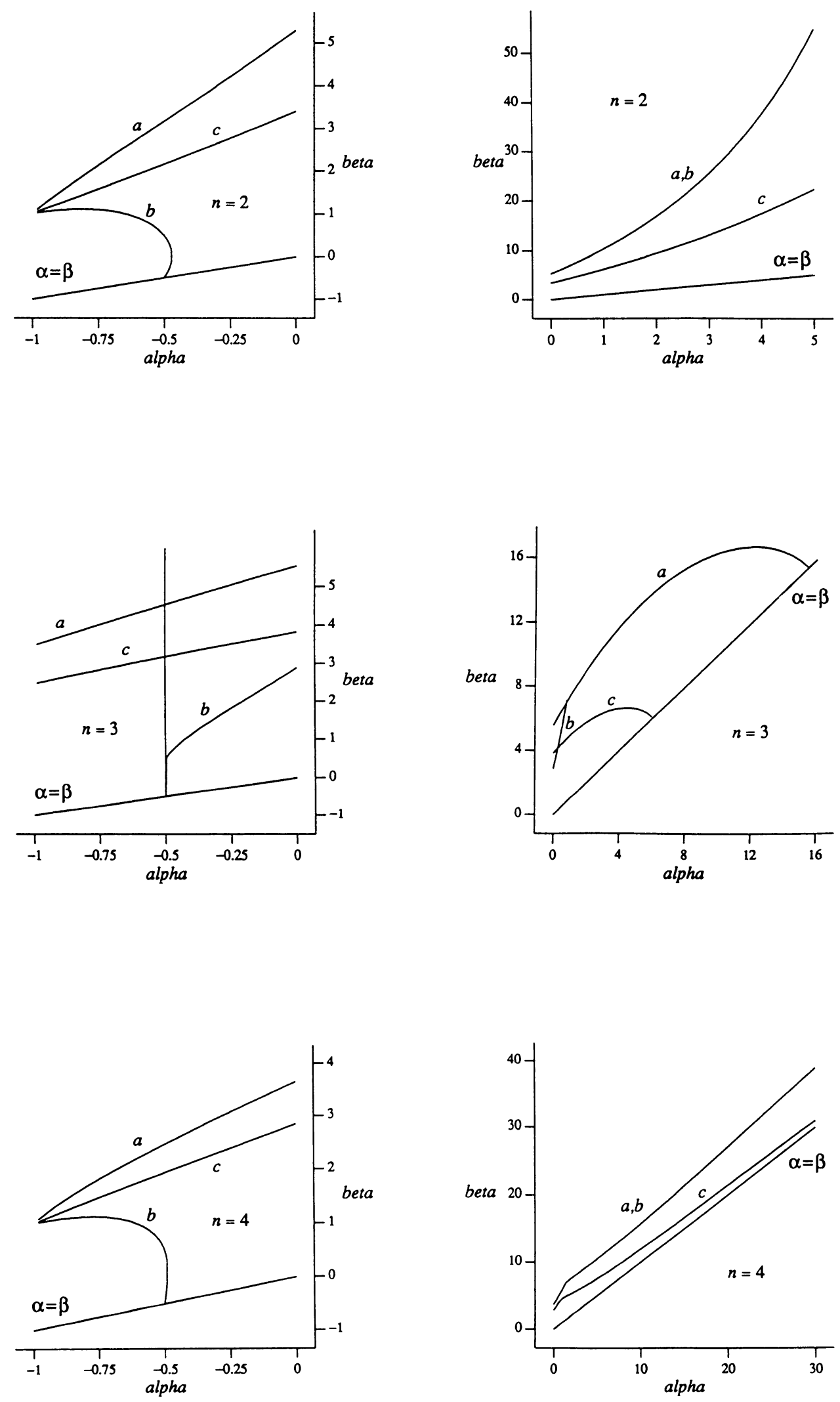

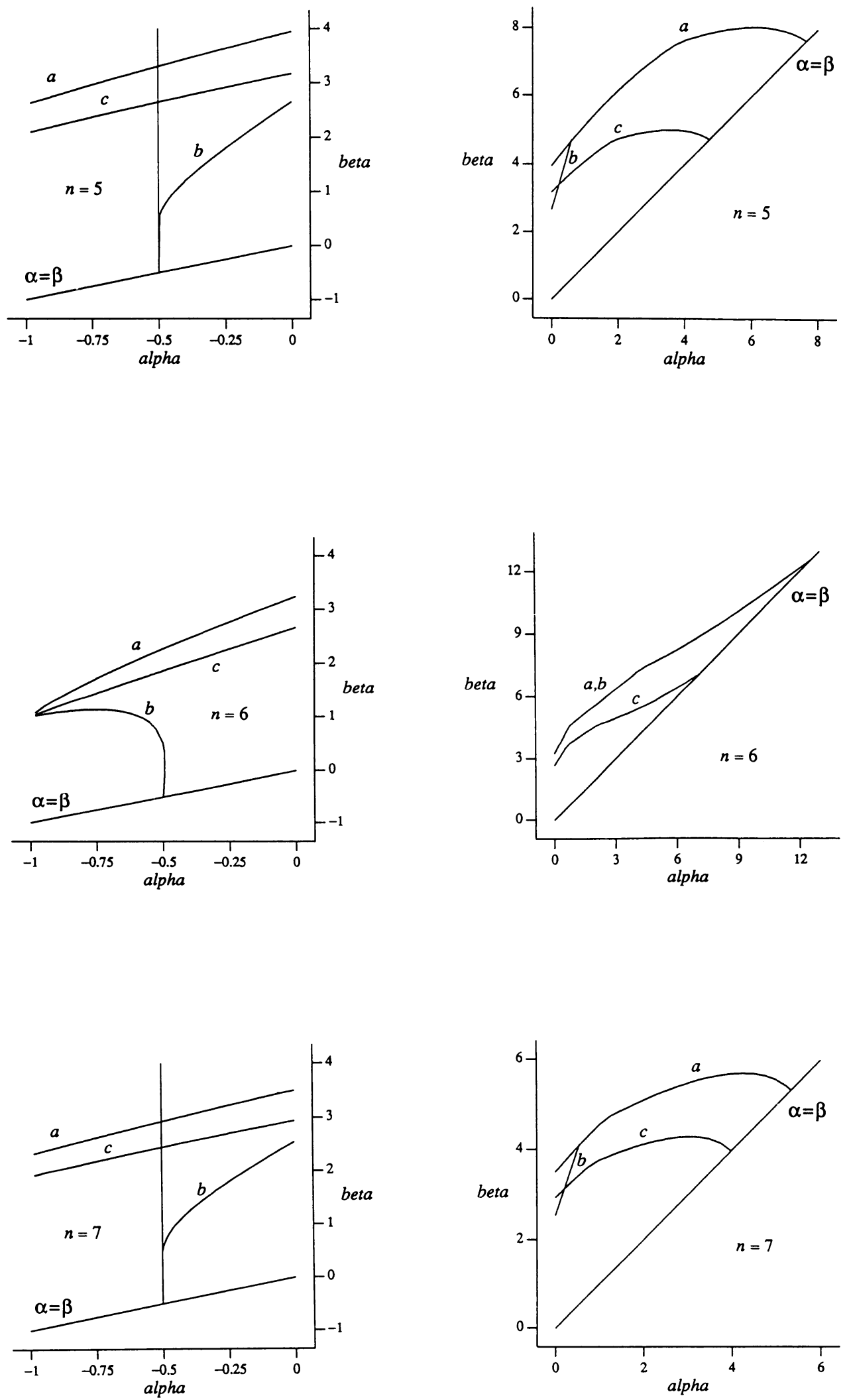

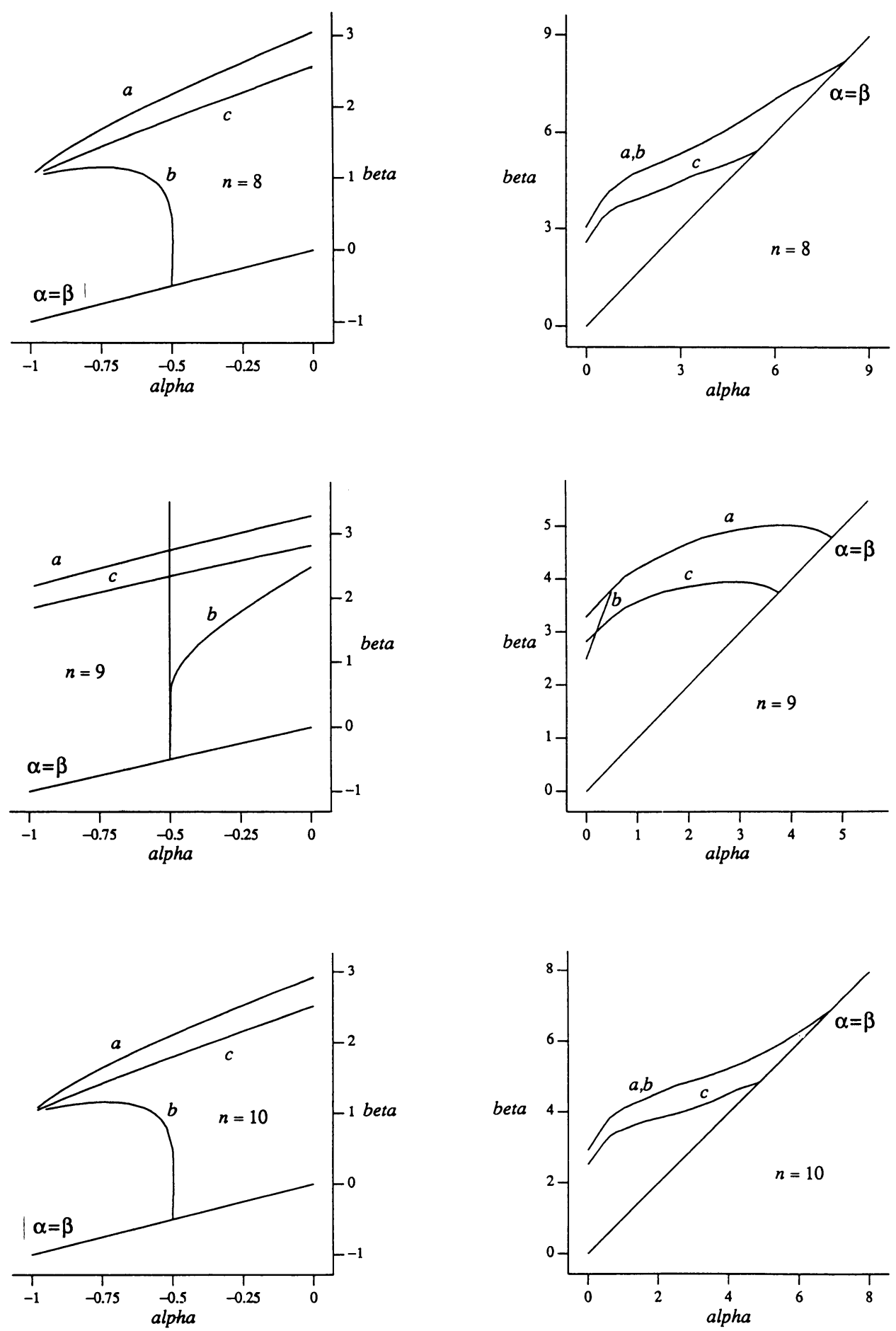

FIGURE 4.1

Property $(p), p=a, b, c$, for the Jacobi weight $w^{(\alpha, \beta)}$. 
By (5.1), the Gauss nodes $\tau_{\nu}=\tau_{\nu}^{(\alpha, 1 / 2)}$ for $\pi_{n}^{(\alpha, 1 / 2)}$ are given by

$$
\tau_{\nu}=2 \bar{\tau}_{\nu}^{2}-1, \quad \nu=1,2, \ldots, n .
$$

Now (5.2) implies (but is not necessarily implied by)

$$
\int_{-1}^{1} t^{2} g\left(t^{2}\right) w^{(\alpha, \alpha)}(t) d t=\sum_{\nu=1}^{2 n+1} \bar{\sigma}_{\nu} \bar{\tau}_{\nu}^{2} g\left(\bar{\tau}_{\nu}^{2}\right)+\sum_{\mu=1}^{2 n+2} \bar{\sigma}_{\mu}^{*} \bar{\tau}_{\mu}^{* 2} g\left(\bar{\tau}_{\mu}^{* 2}\right), \quad \text { all } g \in \mathbf{P}_{3 n+1}
$$

from which, by symmetry,

$$
\int_{0}^{1} t^{2} g\left(t^{2}\right) w^{(\alpha, \alpha)}(t) d t=\sum_{\nu=1}^{n} \bar{\sigma}_{\nu} \bar{\tau}_{\nu}^{2} g\left(\bar{\tau}_{\nu}^{2}\right)+\sum_{\mu=1}^{n+1} \bar{\sigma}_{\mu}^{*} \bar{\tau}_{\mu}^{* 2} g\left(\bar{\tau}_{\mu}^{* 2}\right), \quad \text { all } g \in \mathbf{P}_{3 n+1}
$$

Changing variables, $t==[(\tau+1) / 2]^{1 / 2}$, so that $d t=\frac{1}{4}[(\tau+1) / 2]^{-1 / 2} d \tau$ and $w^{(\alpha, \alpha)}(t)=2^{-\alpha}(1-\tau)^{\alpha}$, yields

$$
\begin{aligned}
& \int_{-1}^{1} g(\tau) w^{(\alpha, 1 / 2)}(\tau) d \tau \\
& =2^{\alpha+5 / 2}\left\{\sum_{\nu=1}^{n} \bar{\sigma}_{\nu} \bar{\tau}_{\nu}^{2} g\left(2 \bar{\tau}_{\nu}^{2}-1\right)+\sum_{\mu=1}^{n+1} \bar{\sigma}_{\mu}^{*} \bar{\tau}_{\mu}^{* 2} g\left(2 \bar{\tau}_{\mu}^{* 2}-1\right)\right\}
\end{aligned}
$$

all $g \in \mathbf{P}_{3 n+1}$.

Since, by (5.4), $2 \bar{\tau}_{\nu}^{2}-1=\tau_{\nu}$ are the Gauss nodes of $w^{(\alpha, 1 / 2)}$, Eq. (5.6) is precisely the (unique) Gauss-Kronrod formula for $w^{(\alpha, 1 / 2)}$ with $n$ Gauss and $n+1$ Kronrod nodes. We have shown:

THEOREM 5.1. The Gauss-Kronrod formula (1.1) for the weight function $w^{(\alpha, 1 / 2)}$ is given by

$$
\begin{array}{ccc}
\tau_{\nu}=2 \bar{\tau}_{\nu}^{2}-1, \quad \sigma_{\nu}=2^{\alpha+5 / 2} \bar{\tau}_{\nu}^{2} \bar{\sigma}_{\nu}, & \nu=1,2, \ldots, n ; \\
\tau_{\mu}^{*}=2 \bar{\tau}_{\mu}^{* 2}-1, \quad \sigma_{\mu}^{*}=2^{\alpha+5 / 2} \bar{\tau}_{\mu}^{* 2} \bar{\sigma}_{\mu}^{*}, & \mu=1,2, \ldots, n+1,
\end{array}
$$

where $\bar{\tau}_{\nu}, \bar{\tau}_{\mu}^{*}$ are the positive nodes in the Gauss-Kronrod formula (5.2) for the weight function $w^{(\alpha, \alpha)}$ and $\bar{\sigma}_{\nu}, \bar{\sigma}_{\mu}^{*}$ the corresponding weights.

Clearly, if the formula (5.2) has property $(p), p=a, b, c, d$, so does formula (5.6). (For property (a), this has previously been observed by Monegato [6, p. 147].) From the discussion in Section 2, we expect this to be true for

$$
\lambda_{2 n+1}^{p}-\frac{1}{2}<\alpha<\Lambda_{2 n+1}^{p}-\frac{1}{2},
$$

so that the Gauss-Kronrod formula (5.6) for the weight $w^{(\alpha, 1 / 2)}$, and hence, by the remark at the beginning of Section 4 , also the one for the weight $w^{(1 / 2, \alpha)}$, has property $(p)$ if (5.9) holds. This means, in particular, that the point

$$
Q_{n}^{p}=\left(\frac{1}{2}, \Lambda_{2 n+1}^{p}-\frac{1}{2}\right)
$$

must lie on or below the curve labeled $p$ in Figure 4.1(n). More precisely, it was observed that for $p=a$ the point $Q_{n}^{a}$ lies strictly below the curve if $n$ is odd, and on the curve if $n$ is even. The reason for this is the phenomenon (2.10) (where $m$ is to be replaced by $m+1$ ) which was observed to hold precisely if $m+1$ (our current 
$n$ ) is odd. Property (a) for (5.2) then ceases to hold because of the collision of a Kronrod node with the Gauss node $\bar{\tau}_{n+1}=0$ at the origin. Since the latter node has no equivalent in the formula (5.6), property (a) continues to hold for (5.6) beyond the critical value $\alpha=\Lambda_{2 n+1}^{a}-\frac{1}{2}$. If $n$ is even, on the other hand, the collision in (5.2) is between a Kronrod node and a nonzero Gauss node, which gives rise to a collision also in (5.6). For the same reason, the point $Q_{n}^{b}$ in (5.10) for property (b) is on the curve labeled $b$ in Figure $4.1(n)$, when $n$ is odd, because of the Kronrod node $\tau_{n+1}^{*}$ becoming equal to -1 , and on the curve labeled $a, b$, when $n$ is even, by virtue of property (a) ceasing to hold. For property (c), $Q_{n}^{c}$ was observed to lie on the curve labeled $c$ in Figure 4.1 $(n)$ when $n$ is even, and below the curve otherwise.

5.2. The weight $|t|^{\gamma}\left(1-t^{2}\right)^{\alpha}$. We now construct the Gauss-Kronrod formula with $2 n+1$ Gauss nodes and $2 n+2$ Kronrod nodes for the weight function

$$
{ }^{\gamma} w^{(\alpha)}(t)=|t|^{\gamma}\left(1-t^{2}\right)^{\alpha} \quad \text { on }-1<t<1, \alpha>-1, \gamma>-1 .
$$

It is known that the associated (monic) orthogonal polynomials are expressible in terms of Jacobi polynomials [4, p. 173]. In particular,

$$
2^{n} \cdot{ }^{\gamma} \pi_{2 n+1}^{(\alpha)}(t)=t \pi_{n}^{(\alpha,(\gamma+1) / 2)}\left(2 t^{2}-1\right) .
$$

Therefore, if $\bar{\tau}_{\nu}, \nu=1,2, \ldots, n$, are the zeros of the Jacobi polynomial $\pi_{n}^{(\alpha,(\gamma+1) / 2)}$, the nodes in the $(2 n+1)$-point Gauss formula for the weight $(5.11)$ are

$$
\begin{gathered}
\tau_{\nu}=\sqrt{\frac{\bar{\tau}_{\nu}+1}{2}}, \quad \nu=1,2, \ldots, n ; \quad \tau_{n+1}=0 ; \\
\tau_{\nu}=-\tau_{2 n+2-\nu}, \quad \nu=n+2, \ldots, 2 n+1 .
\end{gathered}
$$

We now start from the Gauss-Kronrod formula (assumed to exist) for the Jacobi weight $w^{(\alpha,(\gamma+1) / 2)}$,

$$
\int_{-1}^{1} f(t) w^{(\alpha,(\gamma+1) / 2)}(t) d t=\sum_{\nu=1}^{n} \bar{\sigma}_{\nu} f\left(\bar{\tau}_{\nu}\right)+\sum_{\mu=1}^{n+1} \bar{\sigma}_{\mu}^{*} f\left(\bar{\tau}_{\mu}^{*}\right), \quad \text { all } f \in \mathbf{P}_{3 n+1}
$$

Substituting $t=2 \tau^{2}-1$ in the integral on the left yields

$$
\begin{aligned}
& \int_{0}^{1} f\left(2 \tau^{2}-1\right) \tau^{2} \cdot \tau^{\gamma}\left(1-\tau^{2}\right)^{\alpha} d \tau \\
& \quad=2^{-\alpha-(\gamma+5) / 2}\left[\sum_{\nu=1}^{n} \bar{\sigma}_{\nu} f\left(\bar{\tau}_{\nu}\right)+\sum_{\mu=1}^{n+1} \bar{\sigma}_{\mu}^{*} f\left(\bar{\tau}_{\mu}^{*}\right)\right], \quad \text { all } f \in \mathbf{P}_{3 n+1} .
\end{aligned}
$$

Letting $f(u)=[(u+1) / 2]^{k}, k=0,1, \ldots, 3 n+1$, gives

$$
\begin{aligned}
& \int_{0}^{1} \tau^{2 k+2} \cdot \tau^{\gamma}\left(1-\tau^{2}\right)^{\alpha} d \tau \\
&=2^{-\alpha-(\gamma+5) / 2}\left[\sum_{\nu=1}^{n} \frac{\bar{\sigma}_{\nu}}{\left(\bar{\tau}_{\nu}+1\right) / 2}\left(\sqrt{\frac{\bar{\tau}_{\nu}+1}{2}}\right)^{2 k+2}\right. \\
&\left.+\sum_{\mu=1}^{n+1} \frac{\bar{\sigma}_{\mu}^{*}}{\left(\bar{\tau}_{\mu}^{*}+1\right) / 2}\left(\sqrt{\frac{\bar{\tau}_{\mu}^{*}+1}{2}}\right)^{2 k+2}\right], \\
& k=0,1, \ldots, 3 n+1 .
\end{aligned}
$$


Defining, in analogy to (5.12),

$$
\begin{aligned}
\tau_{\mu}^{*}=\sqrt{\frac{\bar{\tau}_{\mu}^{*}+1}{2}}, \quad \mu=1,2, \ldots, n+1 ; \quad \tau_{\mu}^{*}=-\tau_{2 n+3-\mu}^{*} \\
\\
\mu=n+2, \ldots, 2 n+2
\end{aligned}
$$

and letting

$$
\begin{gathered}
\sigma_{\nu}=2^{-\alpha-(\gamma+5) / 2} \frac{\bar{\sigma}_{\nu}}{\left(\bar{\tau}_{\nu}+1\right) / 2}, \quad \nu=1,2, \ldots, n ; \quad \sigma_{n+1}=\lambda ; \\
\sigma_{\nu}=\sigma_{2 n+2-\nu}, \quad \nu=n+2, \ldots, 2 n+1 ;
\end{gathered}
$$

$$
\begin{gathered}
\sigma_{\mu}^{*}=2^{-\alpha-(\gamma+5) / 2} \frac{\bar{\sigma}_{\mu}^{*}}{\left(\bar{\tau}_{\mu}^{*}+1\right) / 2}, \quad \mu=1,2, \ldots, n+1 ; \\
\sigma_{\mu}^{*}=\sigma_{2 n+3-\mu}^{*}, \quad \mu=n+2, \ldots, 2 n+2,
\end{gathered}
$$

where $\lambda$ will be determined shortly, we can write (5.14) equivalently in the form

$$
\int_{-1}^{1} t^{l} \cdot{ }^{\gamma} w^{(\alpha)}(t) d t=\sum_{\nu=1}^{2 n+1} \sigma_{\nu} \tau_{\nu}^{l}+\sum_{\mu=1}^{2 n+2} \sigma_{\mu}^{*} \tau_{\mu}^{* l}, \quad l=1,2, \ldots, 6 n+4
$$

where both sides are zero if $l$ is odd. If we require (5.16) to hold also for $l=0$ (with $\tau_{n+1}^{0}=0^{0}=1$ ), i.e., if $\lambda$ is chosen so that

$$
2 \sum_{\nu=1}^{n} \sigma_{\nu}+\lambda+2 \sum_{\mu=1}^{n+1} \sigma_{\mu}^{*}=\int_{-1}^{1} \gamma w^{(\alpha)}(t) d t
$$

then

$$
\int_{-1}^{1} f(t)^{\gamma} w^{(\alpha)}(t) d t=\sum_{\nu=1}^{2 n+1} \sigma_{\nu} f\left(\tau_{\nu}\right)+\sum_{\mu=1}^{2 n+2} \sigma_{\mu}^{*} f\left(\tau_{\mu}^{*}\right), \quad \text { all } f \in \mathbf{P}_{6 n+4}
$$

is the desired Gauss-Kronrod formula for the weight ${ }^{\gamma} w^{(\alpha)}$. We have shown:

THEOREM 5.2. The Gauss-Kronrod formula for the weight ${ }^{\gamma} w^{(\alpha)}(t)=$ $|t|^{\gamma}\left(1-t^{2}\right)^{\alpha}$ on $(-1,1)$, with $2 n+1$ Gauss nodes and $2 n+2$ Kronrod nodes, is given by (5.18), where the nodes $\tau_{\nu}, \tau_{\mu}^{*}$ are expressible in terms of the nodes $\bar{\tau}_{\nu}, \bar{\tau}_{\mu}^{*}$ in the Gauss-Kronrod formula (5.13) for the Jacobi weight $w^{(\alpha,(\gamma+1) / 2)}$ by means of (5.12), $\left(5.12^{*}\right)$, and similarly, the weights $\sigma_{\nu}, \sigma_{\mu}^{*}$ are expressible in terms of the weights $\bar{\sigma}_{\nu}, \bar{\sigma}_{\mu}^{*}$ in (5.13) by means of $(5.15),\left(5.15^{*}\right), \sigma_{n+1}=\lambda$ being determined by (5.17).

Clearly, if property $(p), p=a, b, d$, holds for the Gauss-Jacobi-Kronrod formula (5.13), it also holds for formula (5.18). Property (c) for (5.13), on the other hand, does not necessarily imply property (c) for (5.18), since the positivity of $\bar{\sigma}_{\nu}, \bar{\sigma}_{\mu}^{*}$, while implying the positivity of all $\sigma_{\nu}, \sigma_{\mu}^{*}$ other than $\sigma_{n+1}$, may or may not imply $\sigma_{n+1}>0$, depending on whether $\lambda$, as obtained from (5.17), is positive or not. 
Appendix. Property $(p)(p=a, b, c, d)$ for $n=5(1) 20(4) 40$.

TABLE A.1

Property $(p)$ for the Gegenbauer weight $w^{(\lambda-1 / 2, \lambda-1 / 2)}\left(\lambda>-\frac{1}{2}\right)$ holds if $\lambda_{n}^{p}<\lambda<\Lambda_{n}^{p}$. This table shows $\Lambda_{n}^{a}, \Lambda_{n}^{c}$ and $\Lambda_{n}^{d}$, as computed by the methods of Section 2. By Conjectures 2.1-2.4, $\lambda_{n}^{a}=\lambda_{n}^{c}=\lambda_{n}^{d}=-\frac{1}{2}, \lambda_{n}^{b}=0, \Lambda_{n}^{b}=\Lambda_{n}^{a}$ for all $n \geq 5$, and $\Lambda_{n}^{d}=\Lambda_{n}^{a}$ whenever $n=4 r-1, r=1,2,3, \ldots$.

\begin{tabular}{rrcc}
\hline$n$ & \multicolumn{1}{c}{$\Lambda_{n}^{a}$} & $\Lambda_{n}^{c}$ & $\Lambda_{n}^{d}$ \\
\hline 5 & 8.1494082801 & 5.2388459015 & 8.1830000561 \\
6 & 13.1085950564 & 7.6571453588 & 13.1107896727 \\
7 & 5.8401376887 & 4.4759114573 & $\Lambda_{7}^{a}$ \\
8 & 8.7386889750 & 5.9524378395 & 8.7555343902 \\
9 & 5.2935342610 & 4.2497937619 & 5.2945466651 \\
10 & 7.3992715320 & 5.3753659922 & 7.4237962746 \\
11 & 4.8531386151 & 4.0481558230 & $\Lambda_{11}^{a}$ \\
12 & 6.1920646523 & 5.0379559112 & 6.1934889120 \\
13 & 4.6542480033 & 3.9519324055 & 4.6543912620 \\
14 & 5.6700664070 & 4.6801034243 & 5.6700700822 \\
15 & 4.4686100363 & 3.8582584626 & $\Lambda_{15}^{a}$ \\
16 & 5.3822674428 & 4.4807122988 & 5.3826940246 \\
17 & 4.3630476637 & 3.8036436813 & 4.3630833901 \\
18 & 5.1865732169 & 4.3552498234 & 5.1873227488 \\
19 & 4.2595630405 & 3.7488473165 & $\Lambda_{19}^{a}$ \\
20 & 4.9631599397 & 4.2700177278 & 4.9632639191 \\
24 & 4.7114083943 & 4.1057723823 & 4.7114508725 \\
28 & 4.5422887809 & 4.0009291994 & 4.5423182352 \\
32 & 4.4137444535 & 3.9286038916 & 4.4137495863 \\
36 & 4.3224901583 & 3.8624938923 & 4.3225046157 \\
40 & 4.2417789470 & 3.8175327957 & 4.2417792595
\end{tabular}

Department of Computer Sciences

Purdue University

West Lafayette, Indiana 47907

Department of Mathematics

Purdue University

West Lafayette, Indiana 47907

1. F. Caliò, W. Gautschi \& E. MARChetti, "On computing Gauss-Kronrod quadrature formulae," Math. Comp., v. 47, 1986, pp. 639-650.

2. J. J. Dongarra, C. B. Moler, J. R. Bunch \& G. W. Stewart, LINPACK Users' Guide, SIAM, Philadelphia, Pa., 1979.

3. W. GAUTSCHI , "A survey of Gauss-Christoffel quadrature formulae," in E. B. Christoffel (P. L. Butzer and F. Fehér, eds.), Birkhäuser, Basel, 1981, pp. 72-147.

4. K. V. LAŠČENOV, "On a class of orthogonal polynomials," Leningrad. Gos. Ped. Inst. Učen. Zap., v. 89, 1953, pp. 167-189. (Russian) 
5. G. Monegato, "A note on extended Gaussian quadrature rules," Math. Comp., v. 30, 1976, pp. 812-817.

6. G. Monegato, "Stieltjes polynomials and related quadrature rules," SIAM Rev., v. 24, 1982, pp. 137-158.

7. L. N. PuOlokaĬNEN, On the Zeros of Orthogonal Polynomials in the Case of a Sign-Variable Weight Function of Special Form, Diploma paper, Leningrad. Gos. Univ., 1964. (Russian)

8. P. RABINOWITZ, "Gauss-Kronrod integration rules for Cauchy principal value integrals," Math. Comp., v. 41, 1983, pp. 63-78.

9. G. Szegö, Orthogonal Polynomials, Amer. Math. Soc. Colloq. Publ., v. 23, 4th ed., Amer. Math. Soc., Providence, R.I., 1975.

10. B: L. VAN DER WAERDEN, Algebra, vol. 1, Ungar, New York, 1970. 